Parallel Processing Letters Vol. 6 No. 4 (1996) 595-598

(C) World Scientific Publishing Company

\title{
AUTHOR INDEX \\ VOLUME 6 (1996)
}

Allen Carruth, J. and Misra, J., Proof of a real-time mutual-exclusion algorithm

Ambroise, D. and Rozoy, B., Marrella: A tool to analyse the graph of states

Armen, C. and Johnson, D.B., Deterministic leader electron on the asynchronous QRQW PRAM

Bekakos, M.P. and Efremides, O.B., A class of systolic tridiagonal linear solvers

Ben-Asher, Y. and Schuster, A., Low Crosstalk address encodings for optical message switching systems

Ben-Asher, Y. and Schuster, A., Time-size tradeoffs for reconfigurable meshes

Bhattacharjee, G.P., see Pal, M.

Breslauer, D. and Hariharan, R., Optimal parallel construction of minimal suffix and factor automata

Cachera, D. and Utard, G., Proving data-parallel programs: A unifying approach

Cai, W., see Lee, B.-S.

Cappello, P., see Scheiman, C.

Chaudhuri, S., Dimopoulos, Y. and Zaroliagis, C.D., On the parallel complexity of acyclic logic programs

Chen, D.Z. and Hu, X.B., Fast and efficient operations on parallel priority queues

Chung, K.-L., Image template matching on reconfigurable meshes

Claver, J.M., Hernandez, V. and Quintana, E.S., Solving discrete-time lyapunov equations for the cholesky factor on a shared memory multiprocessor

Cosnard, M., Editorial note

Cosnard, M., Editorial note

Cosnard, M., Editorial note

Cosnard, M., Editorial note

Destri, G. and Marenzoni, P., Benchmarking lattice-based applications on parallel architectures -

Diáz de Cerio, L., González, A. and Valero-García, M., Communication pipelining in hypercubes

6 (1996) 251-257

6 (1996) 583-594

6 (1996) 247-250

6 (1996) 355-364

6 (1996) 87-100

6 (1996) 231-245

6 (1996) 439-449

6 (1996) 35-44

6 (1996) 491-505

6 (1996) 563-574

6 (1996) 539-550

6 (1996) 223-230

6 (1996) 451-467

6 (1996) 345-353

6 (1996) 365-376

6 (1996) 1-2

6 (1996) 185-186

6 (1996) 297-298

6 (1996) 437-438

6 (1996) 309-320

6 (1996) 507-523

Dikaiakos, M.D., Rogers, A. and Steiglitz, K., Functional algorithm simulation of the fast multipole method: architectural implications
6 (1996) 55-66 
Dimopoulos, Y., see Chaudhuri, S.

Djamegni, C.T. and Tchuente, M., Scheduling of the DAG

6 (1996) $223-230$ associated with pipeline inversion of triangular matrices

Efremides, O.B., see Bekakos, M.P.

Fabrizio Luccio, see Ferragina, P.

Fernandes, R. and Kanevsky, A., On recursive interconnection networks and their extensions

Ferragina, P. and Fabrizio Luccio, Three techniques for parallel maintenance of a minimum spanning tree under batch of updates

Fürer, M. and Raghavachari, B., Parallel edge coloring approximation

Galley, C.N., An $\Omega(\log n-k \log k)$ time linear cost lower bound for the $k$ functions

González, A., see Diáz de Cerio, L.

Grant-Duff, Z.N. and Harrison, P.G., Parallelism via homomorphisms

Grayson, B. and van de Geijn, R., A high performance parallel strassen implementation

Greenwood, G.W., see Gupta, A.K.

$\mathrm{Gu}$, Q.-P. and Peng, S., Fault tolerant routing in hypercubes and star graphs

Gupta, A.K. and Greenwood, G.W., Applications of evolutionary strategies to fine-grained task scheduling

Hariharan, R., see Breslauer, D.

Harrison, P.G., see Grant-Duff, Z.N.

Heng, A., see Lee, B.-S.

Hernandez, V., see Claver, J.M.

Herrmann, C. and Lengauer, C., On the space-time mapping of a class of divide-and-conquer recursions

Hily, A. and Sotteau, D., Gossiping in $d$-dimensional mesh-bus networks

Hu, X.B., see Chen, D.Z.

Iliopoulos, C.S. and Korda, M., Optimal parallel superprimitivity testing for square arrays

JáJá, J. and Ryu, K.W., An optimal randomized parallel algorithm for the single function coarsest partition problem

Jard, C. and Jourdan, G.-V., Incremental transitive dependency tracking in distributed computations

Johnson, D.B., see Armen, C.

Jourdan, G.-V., see Jard, C.

Kanevsky, A., see Fernandes, $R$.

Kim, G. and Yoon, H., Free submesh list strategy: a best fit submesh allocation in mesh connected multicomputers

Korda, M., see Iliopoulos, C.S.

Kwai, D.-M. and Parhami, B., A generalization of hypercubic networks based on their chordal ring structures

Le Guyadec, Y. and Virot, B., Sequential-like proofs of data-parallel programs

Lee, B.-S., Heng, A., Cai, W. and Tan, T.-A., Task scheduling facility for PVM

6 (1996) 13-26

6 (1996) 355-364

6 (1996) 213-222

6 (1996) 377-388

6 (1996) 213-222

6 (1996) 321-329

6 (1996) 195-202

6 (1996) 507-523

6 (1996) 279-295

6 (1996) 3-12

6 (1996) 551-561

6 (1996) $127-136$

6 (1996) 551-561

6 (1996) 35-44

6 (1996) 279-295

6 (1996) 563-574

6 (1996) 365-376

6 (1996) 525-537

6 (1996) 101-113

6 (1996) 451-467

6 (1996) 299-308

6 (1996) 187-193

6 (1996) 427-435

6 (1996) 247-250

6 (1996) 427-435

6 (1996) 377-388

6 (1996) 75-86

6 (1996) 299-308

6 (1996) 469-477

6 (1996) 415-426

6 (1996) 563-574 
Lengaver, C., see Herrmann, C.

Mao, W., see Nicol, D.M.

Marenzoni, P., see Destri, G.

Misra, J., see Allen Carruth, J.

Mizuno, M., Neilsen, M.L. and Raynal, M., An optimistic protocol for a linearizable distribute shared memory system

Neilsen, M.L., see Mizuno, M.

Nicol, D.M. and Mao, W., On bottleneck partitioning of $k$-ary $n$-cubes

Pal, M. and Bhattacharjee, G.P., An optimal parallel algorithm to color an interval graph

Parhami, B., see Kwai, D.-M.

Peng, S., see Gu, Q.-P.

Pombortsis, A.S., see Veglis, A.A.

Quintana, E.S., see Claver, J.M.

Raghavachari, B., see Fürer, M.

Ravishankar, K. and Singh, S., Gossiping on a ring with radios

Raynal, M., see Mizuno, M.

Roch, J.-L. \& Villard, G., Fast parallel computation of the Jordan normal form of matrices

Rogers, A., see Dikaiakos, M.D.

Rozoy, B., see Ambroise, D.

Ryu, K.W., see JáJá, J.

Scheiman, C. and Cappello, P., A processor-time-minimal design for $3 D$ rectilinear mesh algorithms

Schuster, A., see Ben-Asher, Y.

Schuster, A., see Ben-Asher, Y.

Siegelmann, H.T., On NIL: The software constructor of neural networks

Singh, A.K., Bounded timestamps in process networks

Singh, S., see Ravishankar, $\mathrm{K}$.

Sotteau, D., see Hily, A.

Srimani, P.K., Super rotator: Incrementally extensible directed network graph of sublogarithmic diameter

Steiglitz, K., see Dikaiakos, M.D.

Stojmenovic, I., Generating $n$-ary reflected gray codes on a linear array of processors

Tan, T.-A., see Lee, B.-S.

Tchuente, M., see Djamegni, C.T.

Utard, G., see Cachera, D.

Valero-García, M., see Diáz de Cerio, L.

van de Geijn, R., see Grayson, B.

Veglis, A.A. and Pombortsis, A.S., Memory bandwidth analysis of shared-memory multiprocessors using dynamic request rate

Villard, G., see Roch, J.-L.

Virot, B., see Le Guyadec, Y.

Wang, B.-F., A better analysis of Ben-Asher's algorithm for the conditional cartesian product problem

Wu, M.-Y., A parallel scheduling algorithm for tree structured interconnection networks
6 (1996) 525-537

6 (1996) 389-399

6 (1996) 309-320

6 (1996) 251-257

6 (1996) 265-278

6 (1996) 265-278

6 (1996) 389-399

6 (1996) 439-449

6 (1996) 469-477

6 (1996) 127-136

6 (1996) 67-74

6 (1996) 365-376

6 (1996) 321-329

6 (1996) 115-126

6 (1996) 265-278

6 (1996) 203-212

6 (1996) 55-66

6 (1996) 583-594

6 (1996) 187-193

6 (1996) 539-550

6 (1996) $87-100$

6 (1996) 231-245

6 (1996) 575-582

6 (1996) 259-264

6 (1996) 115-126

6 (1996) 101-113

6 (1996) 479-490

6 (1996) 55-66

6 (1996) 27-34

6 (1996) 563-574

6 (1996) 13-26

6 (1996) 491-505

6 (1996) 507-523

6 (1996) 3-12

6 (1996) 67-74

6 (1996) 203-212

6 (1996) 415-426

6 (1996) 331-344

6 (1996) 45-54 
598 Author Index

Xue, J., Generalising the unimodular approach to restructure imperfectly nested loops

Yoon, H., see Kim, G.

Zaroliagis, C.D., see Chaudhuri, S.
6 (1996) $401-414$

6 (1996) 75-86

6 (1996) 223-230 